\title{
UTILISING DEDICATED PEDESTRIAN NETWORKS TO UNDERSTAND THE RELATIONSHIP BETWEEN ACCESSIBILITY AND PEDESTRIAN DENSITY IN ASIAN CITIES
}

\author{
DANIEL M. PEARCE, RYOJI MATSUNAKA, TETSUHARU OBA \& SHOTA YAMAMOTO \\ Department of Urban Management, Kyoto University, Japan
}

\begin{abstract}
As pedestrian networks tie together all transportation modes, their accessibility is crucial to supporting public transportation and fostering more sustainable and liveable cities. Nevertheless, most studies have relied on street networks when calculating pedestrian accessibility. While street networks can substitute for pedestrian networks in some cases, they fail to capture all paths available to pedestrians. Recent studies argue that this may distort our understanding of connectivity, accessibility and consequently the walkability of an area. This study sought to understand the effect of pedestrian network accessibility on segment-scale pedestrian density. To achieve this goal, dedicated pedestrian networks were constructed for two $400 \mathrm{~m}^{2}$ areas in Bangkok and Osaka. In each site, the effect of accessibility variables, namely reach and gravity, were assessed first before controlling for other environmental factors addressing connectivity, transit proximity and path conditions. Measures were evaluated using ArcGIS and the Urban Network Analysis toolbox using data obtained from OpenStreetMap databases and field surveys. The results show that accessibility variables alone can account for up to $33 \%$ and 35\% of observed pedestrian densities in Bangkok and Osaka, respectively. Accessibility to retail land uses is the biggest driver in this relationship. However, when controlling for all variables, only in the denser, more connected Osaka site did accessibility to retail remain significant, helping to explaining up to $45 \%$ of observed pedestrian densities, while in Bangkok, its effect was minimal with proximity to rail transit being the only significant variable.
\end{abstract}

Keywords: built environment, connectivity, dedicated pedestrian networks, OpenStreetMap, pedestrian accessibility, pedestrian density, Urban Network Analysis

\section{INTRODUCTION}

Throughout the world, cities are being confronted with increasing environmental, economic and social challenges that have been attributed to a car-oriented approach to urban planning and design [1], [2]. An increasing reliance on personal vehicles has intensified these challenges further [3]. Nowhere, are the effects felt more than in Asia's large cities that have experienced rapid rates of growth and motorization to become characterized by severe levels of congestion, pollution and deterioration in their pedestrian environments [4]. The cure commonly touted is to encourage walking over private vehicles by creating pedestrian-friendly environments and investing in public transportation [5]. As every trip begins and ends on foot, walking plays an important role in contributing to the overall efficiency of transportation networks enabling multi-modal trips, and by implication, urban accessibility and sustainability.

The role of the built environment in facilitating walking is well-documented [6]-[9]. Previous studies have confirmed that pedestrian activity is influenced by the "3Ds" - density of population and employment, diversity of land use and design of street networks [5], [9], [10]. These were later expanded to "5Ds" to include destination accessibility and distance to transit [10]. Others have distinguished these features as being either macro- or micro-scale features [11]. Macro-scale features refer to characteristics such as population density, land use diversity and street connectivity that are typically measured objectively at the census block, city or regional levels [12]. These features encourage pedestrian activity through improved 
connectivity and accessibility by increasing route options, reducing distance and bringing destinations within closer proximity [9]. This is achieved through mixing land uses, promoting small block sizes and increasing intersection and street densities, among other methods [9], [13]-[16]. Conversely, micro-scale features refer to pedestrian-oriented facilities, including sidewalk width [17] and continuity [18], and aesthetic design qualities [19], [20]. These features are typically measured at a much finer grain either at the street or neighbourhood level, using objective or subjective methods [12]. Research has consistently focused on street connectivity and pedestrian accessibility. These concepts are closely linked and are key to understanding pedestrian activity. To clarify, pedestrian accessibility refers to the ability of pedestrians to reach destinations or opportunities, with respect to a time or distance cost [21]. Naturally, improved connectivity results in improved accessibility by reducing time and distance. This has led to street connectivity often serving as a proxy for pedestrian accessibility to local destinations in pedestrian-oriented studies.

Recent studies have analysed the relationship between pedestrian activity and surrounding environmental factors including residential density, land use mix and access to retail and transit measured at the street-segment level. Many of these studies utilize Space Syntax variables to provide an account of network connectivity and accessibility. Ozbil et al. [11] analysed the effects of accessibility on pedestrian activity in four Istanbul neighbourhoods before considering the impact of connectivity and design variables. Their results indicated that network connectivity, measured with Space Syntax integration and directional reach, is a significant predictor of pedestrian activity. However, across all neighbourhoods, accessibility to different land uses was the strongest predictor accounting for 38\% of observed pedestrian activity. Özer and Kubat [22] reported similar results between the number of commercial and service land uses, integration and pedestrian activity in a separate study conducted in Istanbul. In the USA, Hajrasouliha and Yin [23], utilized structural equation modelling to highlight relationships between measures of street network connectivity and features including - land use mix, job and population density and pedestrian volumes. Focusing on the total standardized effects on pedestrian density, they found that job density had the highest coefficient (0.383), followed by intersection density (0.264), integration (0.178) and land use mix (0.100). While these studies have focused more on explicit measures of network connectivity, others have applied accessibility indices that focus on capturing specific notions of pedestrian accessibility, including pedsheds [21], [24]; pedestrian route directness [25], [26]; and the gravity index [27]. Kang [5] utilized spatial indices to calculate spatial access to various built environment features that relate to the 5Ds and tested their relationship with pedestrian activity. Kang's results show that among spatial accessibility indices, the gravity index model provided the best fit for pedestrian activity. In addition, higher and more direct routes to destinations and access to public transportation had statistically significant positive effects on walking volumes.

While street networks have worked well as a proxy for pedestrian networks in some cases, a growing number of studies say that relying on them leads to distortions in reality, particularly regarding distance and route choices, potentially affecting our understanding of connectivity, accessibility and consequently the walkability of an area [21], [28]-[30]. For example, distance-based measures of accessibility can be underestimated by as much as $7 \%$ in Asian cities [28] and accessibility to land use opportunities underestimated by as much as $40 \%$ in some American neighbourhoods [21]. This is not surprising considering that street networks fail to account for the finer-grained paths available to pedestrians. These studies suggest that pedestrian networks better represent how pedestrians traverse the built environment 
providing a more robust network for analysis. This raises questions regarding the suitability of previous results and the performance of similar measures when calculated on pedestrian networks. Authors such as Cruise et al. [31] have begun analysing the relationship between pedestrian activity, connectivity and other built environment features derived from pedestrian networks with mixed results. Work in this area is limited, and we argue that similar studies have been conducted in different urban contexts outside of Asia that have distinct urban morphologies and walking cultures.

The objectives of this study are three-fold. First, it aims to clarify the relationship between pedestrian network accessibility and segment-scale pedestrian density. We consider the effect of accessibility variables alone, before controlling for other environmental factors known to impact pedestrian density. Second, it aims to further develop understanding of how accessibility and connectivity metrics perform when derived from dedicated pedestrian networks (DPNs) in Asian cities, by including indices that are rarely applied to pedestrian networks. Third, it aims to determine the applicability of crowdsourced point of interest data in deriving segment-level land uses. These data are used in conjunction with accessibility metrics and may prove useful in developing cities where land use data are often limited. In achieving these aims, it is hoped that other researchers will be encouraged to employ DPNs over street network representations in pedestrian-oriented studies.

\section{METHODOLOGY}

The following sections detail data collection procedures, including a description of study sites, DPNs and study variables for addressing research objectives.

\subsection{Study sites}

DPNs were constructed for two study sites located in Bangkok and Osaka. Both cities were chosen to represent different urban forms and levels of urban development as determined by the UN Human Development Index [32] with scores of 0.755 and 0.909 for Bangkok and Osaka, respectively. Each study site consists of a $400 \mathrm{~m}^{2}$ Euclidean pedestrian network centred on a major metro station, corresponding to a 5-minute walking catchment. Metro stations are the focus of this study due to the large number of Asian cities that are investing in rapid transport systems to alleviate issues related to rapid growth and motorization, and for the role that pedestrian environments play in supporting these investments.

The stations selected in each city are as follows: Bangkok - Sukhumvit, and Osaka Namba. These stations represent retail-led urban centres and were selected due to their high levels of ridership and pedestrian amenities. Bangkok - Sukhumvit has the least dense pedestrian network and is characterized by long blocks and cul-de-sacs. It consists of a mixture of sidewalks, shared paths and several walkways and pedestrian bridges that provide limited crossing opportunities across major thoroughfares. Osaka - Namba is far denser and has a more complex pedestrian environment consisting of shorter blocks, public spaces, pedestrian-only paths and a large network of sub-surface paths. Sidewalks line all major streets while narrow streets are typically shared.

\subsection{Dedicated pedestrian networks}

This study employs the standard approach to pedestrian networks where pedestrian paths are represented as links and intersections as nodes (Fig. 1). DPNs consist of all multi-level 

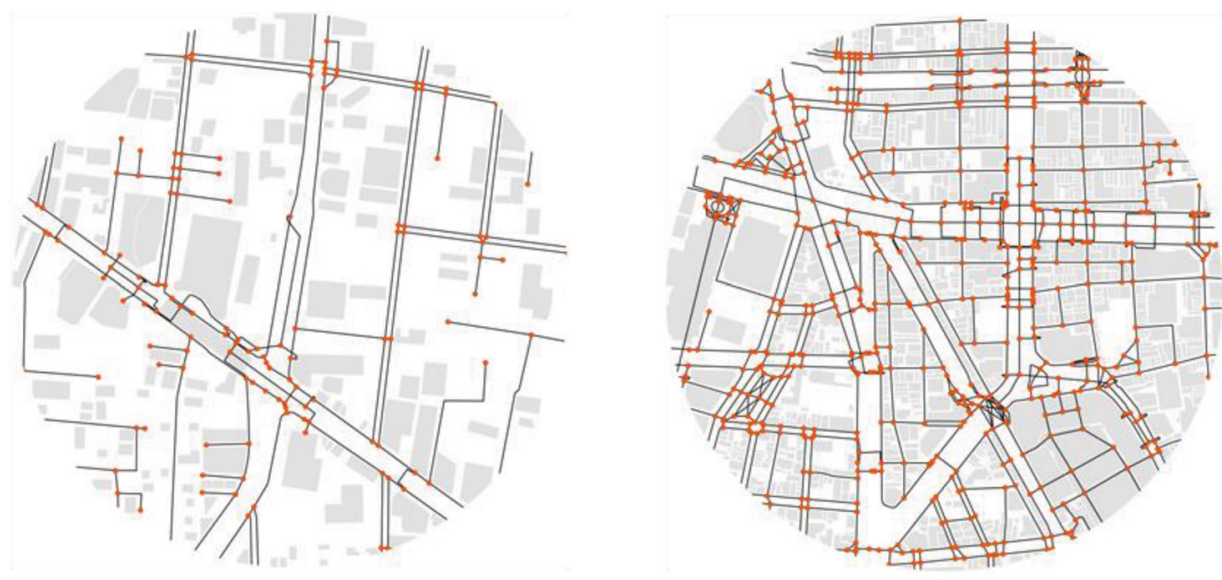

Figure 1: Dedicated pedestrian networks $\left(400 \mathrm{~m}^{2}\right)$.

formal and informal pedestrian facilities that pedestrian have legal access to. Thus, they consist of but are not limited to sidewalks, pedestrian-only zones, shared streets, unmarked crossings and paths through open spaces.

OpenStreetMap (OSM) network data were used to construct geographically accurate DPNs. Network data were cross-referenced with aerial and satellite imagery provided by national agencies or Google Earth services to ensure the integrity of each network link. Missing pedestrian links identified during this process were manually digitized in ArcGIS 10.7 according to the DPN principles detailed by Pearce et al. [28]. These principals were employed to minimize errors when digitizing pedestrian paths to ensure accuracy. Further verification took place during field visits conducted during October to December 2019 to confirm the existence and correctness network links. Errors were further minimized by quality assurance checks and running network connectivity tools within ArcGIS. Finally, each network was used to create a Network Dataset using the ArcGIS Network Analyst extension.

\subsection{Study variables}

This study employs segment-level data in analysing the relationship between accessibility and pedestrian density calculated on DPNs. Common built environment variables were also included to control for their influence on pedestrian density. In total, 11 independent variables were selected - six addressing accessibility to land use opportunities, two addressing connectivity, one transportation proximity and two pedestrian design.

Data were obtained from OSM and field visits conducted to each study site. OSM point of interest (POI) data were used to derive land use destinations for use in conjunction with accessibility metrics. These data consist of point locations that designate destinations ranging from simple roadside objects such as ATMs to large facilities such as retail stores and schools. Land use measures were based on the number of ground-floor frontages at the segment scale [11]. POI data were imported into ArcGIS and then classified into retail/commercial, institutional and leisure land use categories in accordance with the Tokyo Metropolitan 
Government, Bureau of Urban Development [33]. Due to lack of available data, private office and residential uses were excluded. These data were further verified through field visits and by using Google Street View to identify missing data that were manually digitized in ArcGIS. Proportionate stratified random sampling was utilized to select segments for data collection and analysis. Pedestrian counts recorded on each surveyed segment were relativized by the segment's length and multiplied by 100 to give a measure of how many pedestrians were encountered per $100 \mathrm{~m}$ of network [11]. This relativized pedestrian density serves as the dependent variable in this study. Pedestrian counts were conducted during weekdays for two periods: morning (7 am-9 am) and midday (11 am-1 pm).

All variables were calculated using ArcGIS and Urban Network Analyst, an open-source toolbox used to compute centrality measures [34]. Variables were calculated on $400 \mathrm{~m}$ networks unless otherwise stated. Each variable is described as follows:

a) Reach - the cumulative total of each respective land use classification accessible within a $400 \mathrm{~m}$ network distance. Reach is measured on a larger $800 \mathrm{~m}$ network and is defined by eqn (1):

$$
\operatorname{Reach}^{r}[i]=\sum_{j \in G-\{i\}, d[i, j] \leq r} W[j]
$$

where the $\operatorname{Reach}^{r}[i]$ of a node $i$ in a graph $G$ at a search radius $r$ is the number of other nodes in $G$ that are reachable from $i$ at a shortest path distance of at most $r . d[i, j]$ is the shortest path distance between nodes $i$ and $j$, and $\mathrm{W}[j]$ is the weight of node $j$ [34]. In this study, is defined as the midpoint of a surveyed segment, $j$ are destinations or land use opportunities, $r$ is set to $400 \mathrm{~m}$, and the $\mathrm{W}[j]$ is equal to the number of respective land use opportunities for each classification that are accessible at $j$.

b) Gravity - the cumulative total of each respective land use classification accessible within a $400 \mathrm{~m}$ network distance factoring in the spatial impedance required to reach each land use destination. Gravity is measured on a larger $800 \mathrm{~m}$ network and is defined by eqn (2):

$$
\text { Gravity }^{r}[i]=\sum_{j \in G-\{i\}, d[i, j] \leq r} \frac{W[j]}{e^{\beta \cdot d[i, j]}}
$$

where the Gravity ${ }^{r}[i]$ of a node $i$ in a graph $G$ at a search radius $r$ is the number of other nodes in $G$ that are reachable from $i$ at a shortest path distance of at most $r$ [34], and $\beta$ is the spatial impedance value that is set in this study at $0.00217 \mathrm{~m}$, as suggested by Handy and Niemeier [35].

c) Metric Reach - the total network length accessible when walking in all possible directions from each surveyed segment up to a $400 \mathrm{~m}$ radius [36]. It is a measure of network density.

d) Directional Reach - the total network length accessible when walking in all possible directions up to a $400 \mathrm{~m}$ distance with restrictions placed on the number of permitted direction changes specified by a threshold angle [36]. It is a measure of directional connectivity. This study computes directional reach for two turns subject to a ten-degree threshold [16].

e) Distance to Metro - the network distance from each surveyed segment to the nearest metro station entrance. 


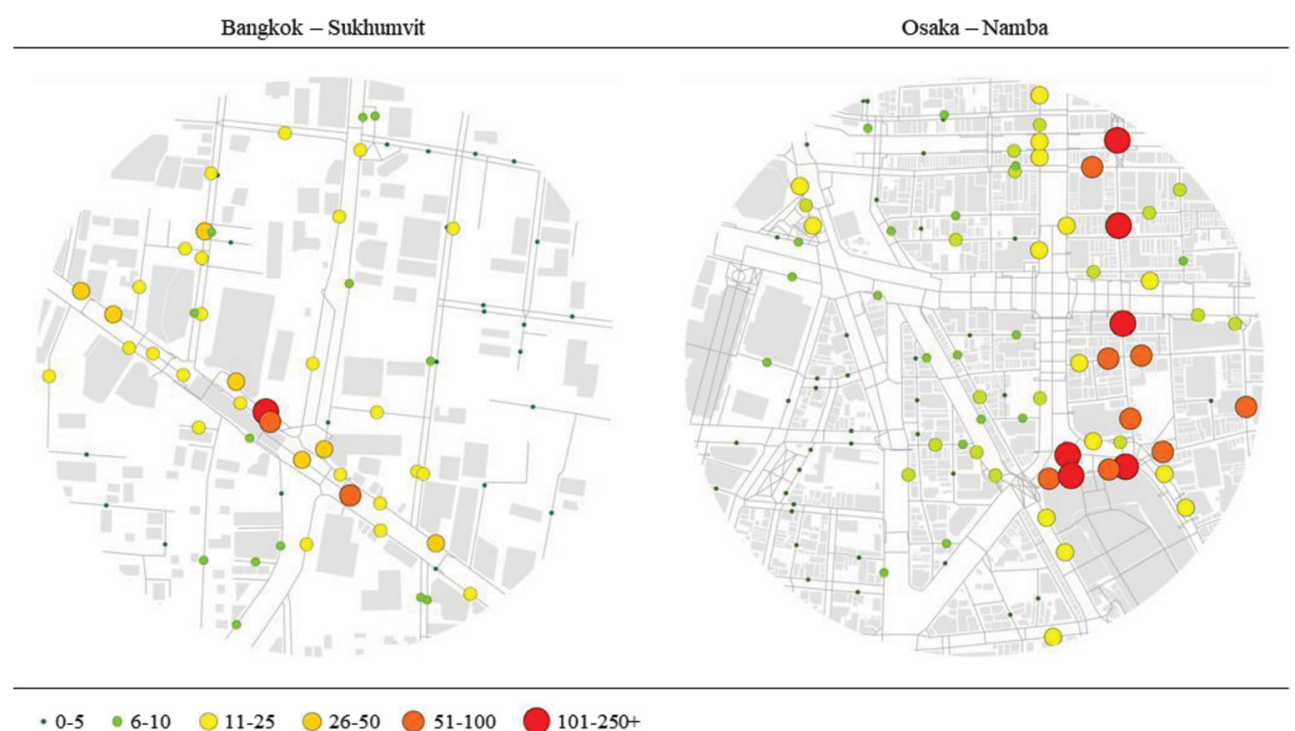

Figure 2: Observed pedestrian densities relativized $\left(400 \mathrm{~m}^{2}\right)$.

f) Path Exclusivity - each surveyed segment is classified as either an exclusive pedestrian path separated from traffic or a shared path. Exclusive paths are a assigned a value of 1 and shared paths are assigned a value of 0 .

g) Path Width - the average width of surveyed segments.

\section{ANALYSING THE EFFECT OF PEDESTRIAN NETWORK ACCESSIBILITY ON PEDESTRIAN DENSITY}

This study investigates the relationship between accessibility, built environment features and segment-level pedestrian density utilizing DPNs. In order to first understand the impact of accessibility metrics, two separate multivariate regression models were created for each study site. The first model referred to as the 'Accessibility' model is composed of only accessibility measures to each of the three land use classifications. The second model factors in all built environmental measures analysed in this study and is referred to as the 'Urban Form' model. Additionally, reach and gravity models are presented separately to avoid collinearity between both indices.

Multivariate regression results for Bangkok are presented in Tables 1 and 2. Focusing first on the reach Accessibility model (Table 1), we can see that accessibility to land use opportunities alone can explain up to $33 \%$ of observed pedestrian densities. Reach accessibility to retail/commercial and institutional land uses have the largest influence on pedestrian density with significant standardized coefficients of 0.332 and 0.258 , respectively. When all variables are introduced in the Urban Form model, the model fit improves to explain up to $44 \%$ of observed pedestrian densities. However, no reach accessibility variables are significant. In this model, pedestrian densities can be overwhelming explained by the distance to the metro station and path exclusivity variables that have significant standardized coefficients of -0.525 and 0.296 , respectively. The explanatory power of the distance to the metro station variable 
Table 1: Bangkok - Sukhumvit: multivariate regression results (reach).

\begin{tabular}{lllllll}
\hline \multirow{2}{*}{ Variable } & \multicolumn{5}{l}{ Accessibility } & \multicolumn{4}{l}{ Urban Form } \\
\cline { 2 - 7 } & $\beta$ & $t$ & Std $\beta$ & $\beta$ & $t$ & Std $\beta$ \\
\hline Reach - Retail/Commercial & 0.004 & 2.687 & $0.332^{* * *}$ & 0.000 & 0.015 & 0.003 \\
Reach - Institutional & 0.131 & 2.023 & $0.258^{* *}$ & -0.026 & -0.311 & -0.052 \\
Reach - Leisure & 0.015 & 0.960 & 0.113 & -0.011 & -0.511 & -0.072 \\
Metric Reach (m) & & & & 0.000 & 0.450 & 0.139 \\
Directional Reach (m) & & & & -0.000 & -0.314 & -0.050 \\
Distance to Metro (m) & & & & -0.011 & -2.556 & $-0.525^{* *}$ \\
Pedestrian Exclusivity & & & & 2.226 & 2.074 & $0.296^{* *}$ \\
Path Width (m) & & & & 0.261 & 1.105 & 0.116 \\
\# Observations: 72 & & & & $0.501 * * *$ & \\
R-Squared & $0.355^{* * *}$ & & $0.438^{* * * *}$ & \\
Adj. R-Squared & $0.327^{* * *}$ & & &
\end{tabular}

$* p<0.10 ; * * p<0.05 ; * * * p<0.01$

Table 2: Bangkok - Sukhumvit: multivariate regression results (gravity).

\begin{tabular}{|c|c|c|c|c|c|c|}
\hline \multirow{2}{*}{ Variable } & \multicolumn{3}{|c|}{ Accessibility } & \multicolumn{3}{|c|}{ Urban Form } \\
\hline & $\beta$ & $t$ & $\operatorname{Std} \beta$ & $\beta$ & $t$ & $\operatorname{Std} \beta$ \\
\hline Gravity - Retail/Commercial & 0.006 & 2.345 & $0.302 * *$ & -0.002 & -0.511 & -0.091 \\
\hline Gravity - Institutional & 0.215 & 1.844 & 0.248 & -0.078 & -0.506 & -0.090 \\
\hline Gravity - Leisure & 0.033 & 1.110 & 0.133 & -0.017 & -0.490 & -0.072 \\
\hline Metric Reach (m) & & & & 0.001 & 0.645 & 0.213 \\
\hline Directional Reach (m) & & & & -0.000 & -0.458 & -0.073 \\
\hline Distance to Metro (m) & & & & -0.011 & -2.839 & $-0.593 * *$ \\
\hline Pedestrian Exclusivity & & & & 2.048 & 1.919 & $0.273^{*}$ \\
\hline Path Width (m) & & & & 0.247 & 1.058 & 0.110 \\
\hline \multicolumn{7}{|l|}{ \# Observations: 72} \\
\hline R-Squared & \multicolumn{3}{|l|}{$0.339 * * *$} & \multicolumn{3}{|l|}{$0.504 * * *$} \\
\hline Adj. R-Squared & \multicolumn{3}{|l|}{$0.309 * * *$} & \multicolumn{3}{|c|}{$0.441 * * *$} \\
\hline
\end{tabular}

$* p<0.10 ; * * p<0.05 ; * * * p<0.01$

can be explained largely by network structure. As distance increases from the station, the network paths become less connected consisting of many cul-de-sacs. Thus, pedestrians are not found in as high volumes in these locations further from the station.

Similar results are observed when we replace reach with gravity in the models (Table 2). First, the reach Accessibility model produces a higher adjusted $\mathrm{R}^{2}$ explaining $33 \%$ of observed pedestrian densities versus $31 \%$ for the gravity Accessibility model. Additionally, only gravity 
accessibility to retail/commercial land uses remains significant. This suggests that when 400 $\mathrm{m}$ is used as the search radius to compute reach and gravity, the spatial impedance factor included in the calculation of gravity marginally reduces the attractiveness of destinations, making it slightly less effective in explaining pedestrian density. When all variables are introduced in the Urban Form model, the model fit remains more or less the same whether or not accessibility is measured with the reach or gravity index. The poor performance of both reach accessibility and gravity accessibility in the Urban Form models is likely explained by the relatively high correlations observed between accessibility measures and distance to the metro station. The land uses examined in this study have a tendency to accumulate around the metro station where several large shopping centres are located and decrease with distance from the station.

Multivariate regression results for Osaka are presented in Tables 3 and 4. Focusing first on the reach Accessibility model (Table 3), we can see that accessibility to land use opportunities alone can explain up to $34 \%$ of observed pedestrian densities. Reach accessibility to retail/commercial and leisure land uses have the largest influence on pedestrian density with statistically significant standardized coefficients of 0.529 and 0.247 , respectively. When all variables are introduced in the Urban Form model, the model fit improves to explain up to $45 \%$ of observed pedestrian densities - a similar magnitude of improvement observed in the corresponding Bangkok models. In contrast to Bangkok, all reach accessibility variables are statistically significant, while distance to the metro station is no longer significant. Additionally, pedestrian densities are explained by a variety of variables including accessibility, metric reach and path width. The most important variables in the Urban Form model are reach accessibility to retail/commercial and leisure land uses, and pedestrian path exclusivity with significant standardized coefficients of $0.344,0.263$ and 0.234 , respectively.

Replacing reach with gravity in the Accessibility models produces similar results (Table 4). This time the gravity Accessibility model produces a higher adjusted $\mathrm{R}^{2}$ explaining $35 \%$ of observed pedestrian densities versus $34 \%$ for the reach Accessibility model. However, this difference is

Table 3: Osaka - Namba: multivariate regression results (reach).

\begin{tabular}{lllllll}
\hline \multirow{2}{*}{ Variable } & \multicolumn{4}{l}{ Accessibility } & \multicolumn{4}{l}{ Urban Form } \\
\cline { 2 - 7 } & $\beta$ & $t$ & Std $\beta$ & $\beta$ & $t$ & Std $\beta$ \\
\hline Reach - Retail/Commercial & 0.002 & 5.932 & $0.529^{* * *}$ & 0.001 & 3.526 & $0.344^{* * *}$ \\
Reach - Institutional & -0.052 & -1.284 & -0.134 & -0.081 & -1.994 & $-0.208^{* *}$ \\
Reach - Leisure & 0.003 & 2.429 & $0.247^{* *}$ & 0.003 & 2.326 & $0.263^{* *}$ \\
Metric Reach (m) & & & & 0.000 & 0.765 & $0.229^{* *}$ \\
Directional Reach (m) & & & & 0.000 & 0.765 & 0.073 \\
Distance to Metro (m) & & & & -0.002 & -0.478 & -0.049 \\
Pedestrian Exclusivity & & & & 1.727 & 2.625 & $0.234^{* *}$ \\
Path Width (m) & & & & 0.133 & 1.974 & $0.155^{*}$ \\
\# Observations: 105 & & & & $0.488^{* * *}$ & & \\
R-Squared & $0.356^{* * *}$ & & $0.446^{* * *}$ & & \\
Adj. R-Squared & $0.337^{* * *}$ & & & & &
\end{tabular}

$* p<0.10 ; * * p<0.05 ; * * * p<0.01$ 
Table 4: Osaka - Namba: multivariate regression results (gravity).

\begin{tabular}{|c|c|c|c|c|c|c|}
\hline \multirow{2}{*}{ Variable } & \multicolumn{3}{|c|}{ Accessibility } & \multicolumn{3}{|c|}{ Urban Form } \\
\hline & $\beta$ & $t$ & Std $\beta$ & $\beta$ & $t$ & $\operatorname{Std} \beta$ \\
\hline Gravity - Retail/Commercial & 0.004 & 6.187 & $0.537 * * *$ & 0.002 & 3.445 & $0.336 * * *$ \\
\hline Gravity - Institutional & -0.098 & -1.631 & -0.164 & -0.132 & -2.164 & $-0.220 * *$ \\
\hline Gravity - Leisure & 0.006 & 2.611 & $0.261 * *$ & 0.007 & 2.501 & $0.274 * *$ \\
\hline Metric Reach (m) & & & & 0.001 & 2.162 & $0.237 * *$ \\
\hline Directional Reach (m) & & & & 0.000 & 0.840 & 0.080 \\
\hline Distance to Metro (m) & & & & -0.002 & -0.407 & -0.042 \\
\hline Pedestrian Exclusivity & & & & 1.610 & 2.440 & $0.218^{* *}$ \\
\hline Path Width (m) & & & & 0.123 & 1.828 & $0.143^{*}$ \\
\hline \multicolumn{7}{|l|}{ \# Observations: 105} \\
\hline R-Squared & $0.366^{* * *}$ & & & $0.488 * *$ & & \\
\hline Adj. R-Squared & $0.347 * * *$ & & & $0.445^{* * *}$ & & \\
\hline
\end{tabular}

$* p<0.10 ; * * p<0.05 ; * * * p<0.01$

marginal and the standardized regression coefficients for reach and gravity accessibility variables are similar. When all variables are introduced in the Urban Form model, the model fit remains more or less the same whether or not accessibility is measured with the reach or gravity index, and a similar magnitude of improvement is observed in the corresponding Bangkok models. The strength of standardized regression coefficients and their significant levels also remains very similar whether accessibility is measured with reach or gravity.

\section{DISCUSSION}

Our findings offer insights into the comparative roles of accessibility and environmental factors in influencing pedestrian activity when utilizing DPN in Asian cities. Focusing solely on accessibility to land use opportunities alone, our results show that accessibility variables can account for up to $35 \%$ of observed pedestrian densities. Across both study sites, it is accessibility to retail/commercial land uses that underpins this relationship. When controlling for built environment variables, only in the denser, more connected Osaka site did accessibility to retail/commercial land uses remain significant, helping to explain up to $45 \%$ of observed pedestrian densities. While in Bangkok, its effect was minimal with proximity to rail transit being the most significant variable.

Fundamentally, the pedestrian environments in both study sites are different. In Osaka, retail/commercial and leisure land uses are more dispersed throughout and are greater in number. In contrast, these land uses in Bangkok are located closer to the station. This is best exemplified by the pedestrian shopping streets spread throughout the Osaka site, compared to department stores located at the station in Bangkok that account for the majority of retail/commercial land uses. Pedestrian network forms also differ between study sites and likely had a major impact on our findings. For example, the pedestrian environment in Osaka is more complex and connected with many sub-surface paths. This allows easier access to land use opportunities. In contrast, the Bangkok pedestrian environment is less connected 
with many dead ends. Finally, a key difference between both study sites was the power of rail transit proximity in explaining pedestrian density. Osaka has clearly invested heavily in pedestrian access to their metro stations. As there are many entrances, the station's power of attraction is effectively nullified as the distance to the station from each surveyed segment remains more or less constant throughout the entire study site. This contrasts the situation in Bangkok where the entrances are smaller in number and concentrated at the centre of the site.

\subsection{Challenges and further study}

The quality and availability of data vary significantly from country to country. To counteract this, we utilized crowdsourced data from OSM to help create DPNs and derive land use measures. While OSM network data is helpful in creating pedestrian networks, it is still a time-consuming process that relies on a lot of manual verification. Alternative methods should be explored that will allow more study sites to be included in analysis. Larger pedestrian networks should also be investigated. In this study, while the focus was on $400 \mathrm{~m}^{2}$ networks, larger networks should be analysed to account for different walking distances, which may provide stronger results. This will allow more accurate conclusions to be drawn on the relationship between accessibility measures calculated on DPNS and the applicability of these networks themselves. Additionally, while this study proved that land use data derived from points of interest can be a good substitute for more detailed datasets, it is still less than ideal. Data that incorporate vertical land uses or building-level data would likely provide more robust results. Data availability issues also resulted in key variables being omitted from the analysis. For example, employment or population density data would likely have had a large impact on pedestrian density in these environments. Nevertheless, our methods have the potential to be applied particularly to developing cities where data are scare.

It is important to note that accessibility measures calculated on pedestrian networks, speaks nothing to the configurational or structural ability of networks themselves in influencing pedestrian activity. This study forms part of larger doctoral research project exploring the relationship between pedestrian network centrality, micro-scale pedestrian environments and pedestrian activity in five Asian cities. This research will help to create a clearer picture of what factors influence pedestrian activity at the segment-scale in Asia and will hopefully make a strong case for using DPNs in pedestrian-oriented studies. Finally, our results strengthen the argument that in order to encourage pedestrian activity and create livelier, more walkable environments, policies should focus on improving accessibility to retail/commercial land uses in these environments.

\section{ACKNOWLEDGEMENTS}

None.

\section{REFERENCES}

[1] Speck, J., Walkable City: How Downtown Can Save America One Step at a Time, Farrar, Straus and Giroux: New York, 2012.

[2] Frumkin, H., Frank, L. \& Jackson, R.J., Urban Sprawl and Public Health: Design, Planning, and Building Health Communities, Island Press: Washington DC, 2004.

[3] Davis, S.C., Diegel, S.W. \& Boundy, R.G., Transportation Energy Data Book: Edition 35, Oak Ridge National Laboratory, 6992, 2016. 
[4] Leather, J., Fabian, H., Gota, S. \& Mejia, A., Walkability and Pedestrian Facilities in Asian Cities: State and Issue, Asian Development Bank, 17, 2011.

[5] Kang, C.-D., The S + 5Ds: Spatial access to pedestrian environments and walking in Seoul, Korea, Cities, 77, pp. 130-141, 2018.

[6] Brownson, R.C., Hoehner, C.M., Day, K., Forsyth, A. \& Sallis, J.F., Measuring the built environment for physical activity: state of the science. American Journal of Preventive Medicine, 36(4), pp. 99-123, 2009.

[7] Saelens, B.E. \& Handy, S., Built environment correlates of walking: a review. Medicine and Science in Sports and Exercise, 40(7), pp. 550-566, 2008.

[8] Bauman, A.E. \& Bull, F.C., Environmental Correlates of Physical Activity and Walking in Adults and Children: A Review of Reviews, National Institute for Health and Care Excellence (NICE): Loughborough, 2007.

[9] Cervero, R. \& Kockelman, K., Travel demand and the 3Ds: density, diversity, and design. Transportation Research Part D: Transport and Environment, 2(3), pp. 199-219, 1997.

[10] Ewing, R. \& Cervero, R., Travel and the built environment. Journal of the American Planning Association, 76(3), pp. 265-294, 2010.

[11] Ozbil, A., Gurleyen, T., Yesiltepe, D. \& Zunbuloglu, E., Comparative associations of street network design, streetscape attributes and land-use characteristics on pedestrian flows in peripheral neighbourhoods. International Journal of Environmental Research and Public Health, 16(1846), pp. 1-23, 2019.

[12] Nawrocki, J., Nakagawa, D., Matsunaka, R. \& Oba, T., Measuring walkability and its effect on light rail usage: a comparative study of the USA and Japan. WIT Transactions on the Built Environment, 138, pp. 305-316, 2014.

[13] Hess, P., Moudon, A., Snyder, M. \& Stanilov, K., Site design and pedestrian travel. Transportation Research Record, 1674(1), pp. 9-19, 1999.

[14] Handy, S. \& Clifton, K., Evaluating neighborhood accessibility: possibilities and practicalities. Journal of Transportation Statistics, 4(2), pp. 67-78, 2001.

[15] Kerr, J., Frank, L., Sallis J.F. \& Chapman, J., Urban form correlates of pedestrian travel in youth: differences by gender, race-ethnicity and household attributes. Transportation Research Part D: Transport and Environment, 12(3), pp. 177-182, 2007.

[16] Ozbil, A., Peponis, J. \& Stone, B., Understanding the link between street connectivity, land use and pedestrian flows. Urban Design International, 16(2), pp. 125-141, 2011.

[17] Duncan, M.J., Spence, J.C. \& Mummery, W.K., Perceived environment and physical activity: a meta-analysis of selected environmental characteristics. International Journal of Behavioral Nutrition and Physical Activity, 2(11), pp. 1-9, 2005.

[18] Evenson, K.R., Sotres-Alvarez, D., Herring, A.H., Messer, L., Laraia, B.A. \& Rodríguez, D.A., Assessing urban and rural neighborhood characteristics using audit and GIS data: derivation and reliability of constructs. International Journal of Behavioral Nutrition and Physical Activity, 6(44), pp. 1-16, 2009.

[19] Mitra, R., Siva, H. \& Kehler, M., Walk-friendly suburbs for older adults? Exploring the enablers and barriers to walking in a large suburban municipality in Canada. Journal of Aging Studies, 35, pp. 10-19, 2015.

[20] Ewing et al., Validating urban design measures. Transportation Research Board $92^{\text {nd }}$ Annual Meeting, Washington DC, 2013.

[21] Tal, G. \& Handy, S., Measuring non-motorized accessibility and connectivity in a robust pedestrian network. Transportation Research Record: Journal for Transportation Research Board, 2299(1), pp. 48-56, 2012. 
[22] Ozer, O. \& Kubat, A.S., Walking initiatives: a quantitative movement analysis. Proceedings, 6th International Space Syntax Symposium, Istanbul, pp. 1-16, 2007.

[23] Hajrasouliha, A. \& Yin, L., The impact of street network connectivity on pedestrian volume. Urban Studies, 52(13), pp. 2483-2497, 2014.

[24] Porta, S. \& Renne, J.L. Linking urban design to sustainability: formal indicators of social urban sustainability field research in Perth, Western Australia. Urban Design International, 10(1), pp. 51-64, 2005.

[25] Randall, T.A. \& Baetz, B.W., Evaluating pedestrian connectivity for suburban sustainability. Journal of Urban Planning and Development, 127(1), pp. 1-15, 2001.

[26] Hess, P.M., Measures of connectivity [streets: old paradigm, new investment]. Places, 11(2), pp. 58-65, 1997.

[27] Hansen, W.G., How accessibility shapes land use. Journal of the American Institute of Planners, 25(2), pp. 73-76 1959.

[28] Pearce, D.M., Matsunaka, R. \& Oba, T., Comparing accessibility and connectivity metrics derived from dedicated pedestrian networks and street networks in the context of Asian cities. Asian Transport Studies, 7, pp. 1-10, 2021.

[29] Zhang, H. \& Zhang, Y., Pedestrian network analysis using a network consisting of formal pedestrian facilities: sidewalks and crosswalks. Transportation Research Record: Journal of the Transportation Research Board, 2673(7), pp. 294-307, 2019.

[30] Ellis, G., Hunter, R., Tully, M.A., Donnelly, M., Kelleher, L. \& Kee, F., Connectivity and physical activity: using footpath networks to measure the walkability of built environments. Environmental and Planning B: Planning and Design, 43(1), pp. 130 $151,2016$.

[31] Cruise, S.M., Hunter, R.F., Kee, F., Donnelly, M., Ellis, G. \& Tully, M.A., A comparison of road- and footpath-based walkability indices and their associations with active travel. Journal of Transport \& Health, 6, pp. 119-127, 2017.

[32] United Nations Development Programme. Human Development Indices and Indicators: 2018 Statistical, New York, 2018.

[33] Tokyo Metropolitan Government, Department of Urban Development, Land Use in Tokyo: Overview of Land Use Survey for FY2012, 2012. In Japanese.

[34] Sevtsuk, A., \& Mekonnen, M., Urban network analysis. A new toolbox for ArcGIS. Revue Internationale de Géomatique, 22(2), pp. 287-305, 2012.

[35] Handy, S.L. \& Niemeier, D.A., Measuring accessibility: an exploration of issues and alternatives. Environment and Planning A: Economy and Space, 29(7), pp. 1175-119, 1997.

[36] Peponis, J., Bafna, S. \& Zhang, Z. The connectivity of streets: reach and directional distance. Environment and Planning B: Planning and Design, 35(5), pp. 881-901, 2008. 